\title{
Efectos del reimplante vesicoureteral en pacientes con trasplante renal y con reflujo vesicoureteral del injerto en la clínica universitaria Colombia, 2014-2016
}

\section{Outcomes of Ureteroneocystostomy for Vesicoureteral Reflux in Patients with Renal Transplant, in a Referral Center in Colombia 2014-2016}

\author{
Nataly González González ${ }^{1}$ \\ Milena Rodríguez Bedoya ${ }^{3}$ \\ ${ }^{1}$ Fundación Universitaria Sanitas, Bogotá, Colombia \\ ${ }^{2}$ Grupo de Trasplante Renal, Clínica Universitaria Colombia Bogotá, \\ Bogotá, Colombia \\ ${ }^{3}$ Departmento de Epidemiología Clínica, Fundación Universitaria \\ Sanitas Bogotá, Bogotá, Colombia
}

Ana Carolina Lizcano Herrera ${ }^{1}$

Address for correspondence Nataly González, MD, Calle 23 No 66- 46 Clínica Universitaria Colombia, Bogotá, Colombia

(e-mail: natalygonzalez_1@hotmail.com).

Urol Colomb 2018;27:272-276.

\section{Resumen}

Palabras Clave

- trasplante renal

- complicaciones postoperatorias

- reflujo vesicoureteral

- infección recurrente

- infección tracto urinario

- insuficiencia renal
Introducción y Objetivos El reflujo vesicoureteral posterior al trasplante renal es una complicación poco frecuente. Esa patología favorece las infecciones urinarias y el deterioro de la función renal, dependiendo de las características y del grado de reflujo, se puede realizar el tratamiento. Sin embargo, el manejo definitivo consiste en la reintervención quirúrgica ya sea por vía endoscópica o abierta. No se tiene conocimiento sobre las características clínicas, así como la función renal y la prevalencia de infecciones urinarias en esa población. El objetivo de este trabajo, fue describir las principales características clínicas relacionadas con la función renal pre y post reimplante ureteral y la prevalencia de infecciones urinarias y hospitalizaciones en pacientes trasplantados renales con reflujo vesicoureteral.

Materiales y Métodos Estudio descriptivo de serie de expuestos, realizado en pacientes trasplantados renales con reflujo vesicoureteral sometidos a ureteroneocistotomía. Las principales variables de estudio fueron función renal, número de infecciones urinarias y necesidad de hospitalización, previo al reimplante ureteral y a los tres meses posteriores. Se tomó la totalidad de pacientes operados entre los años 2014 y 2016. Se realizó un análisis descriptivo con medidas de frecuencia y tendencia central y una comparación de los resultados clínicos según la técnica quirúrgica usada (abierta o endoscópica).

Resultados en el estudio se incluyeron 16 pacientes trasplantados renales con reflujo vesicoureteral del injerto como complicación postoperatoria que posteriormente fueron sometidos a la ureteroneocistostomía. El promedio de edad de la población received

October 18, 2016

accepted

March 9, 2018

published online

July 24,2018
DOI https://doi.org/

$10.1055 / \mathrm{s}-0038-1645842$

ISSN 0120-789X.

eISSN 2027-0119.
Copyright $\odot$ 2018, Sociedad Colombiana License terms de Urología. Publicado por Thieme Revinter Publicações Ltda., Rio de Janeiro, Brazil. Todos los derechos reservados.

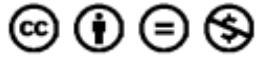


fue de 43 años (D.E. \pm 14,5), trasplante renal con donante cadavérico en un 87,5\% y vivo del $12,5 \%$. Según el grado de reflujo vesicoureteral previo a la corrección, el 41,6\% eran grado III, seguido por grado II en el $25 \%$ y grado IV en el $25 \%$. El abordaje quirúrgico de elección para el reimplante fue abierto en un $68,7 \%$ y endoscópico en el $31,2 \%$ de los casos. La función renal posterior al reimplante muestra tendencia a la mejoría con un promedio en el nivel de creatinina pre en 1.66 (D.E. $\pm 1,19$ ) y post reimplante ureteral en 1.5 (D.E. \pm 0,64). Por otra parte, las infecciones urinarias disminuyeron de 4.5 (D. E. $\pm 3,2$ ) a 1.1 (D.E. $\pm 1,36$ ) por año y el número de hospitalizaciones se redujo de 2.8 (D.E. $\pm 3,2$ ) a 0.9 (D.E. $\pm 1,28$ ) en el seguimiento.

Conclusiones en los pacientes con trasplante renal y corrección quirúrgica del reflujo vesicoureteral se encontraron niveles estables de azoados y se observó una disminución en el número de infecciones urinarias y de necesidad de hospitalizaciones, lo cual puede servir de base para futuros estudios con mayor muestra, analíticos y prospectivos que demuestren el impacto del reimplante vesicoureteral en la función renal y en las infecciones urinarias en ese grupo de pacientes.

Abstract

\section{Keywords}

- renal transplantation

- postoperative complications

- vesicoureteral reflux

- recurrent infection

- urinary tract infection

- renal failure
Introduction and Objectives Vesicoureteral reflux after renal transplantation is a rare complication, this condition favors urinary tract infections and deterioration of renal function. Treatment depends on the characteristics and degree of reflux; however, the definitive management consists in surgical reintervention, either open or endoscopically. There is a lack of evidence until now about the prevalence of urinary tract infections and renal function in this population. The objective of this study is to describe the clinical characteristics related to renal function, and clinically significant events, before and after ureteral reimplantation in renal transplant patients with vesicoureteral reflux.

Materials and Methods Descriptive study of a case series. We included renal transplant patients with vesicoureteral reflux in whom ureteroneocystotomy was performed. We assessed renal function with serum creatinine, number of episodes of urinary tract infections and need for in-patient care prior and three months after surgery, between 2014 and 2016. Analysis was made with descriptive statistics and a subgroup evaluation considering the surgical technique (open or endoscopic).

Results We included 16 patients. The average age of the population was 43 years (SD 14.5), $87.5 \%$ of the patients had a cadaverous donor and $12.5 \%$ live donor. $41.6 \%$ had grade III vesicoureteral reflux, $25 \%$ grade II and grade IV. $68.7 \%$ had an open surgical approach, the remaining $31.2 \%$ were done endoscopically. Renal function tend to improve (sCr pretransplant 1.66 SD 1.19 versus sCr posttransplant 1.5 SD 0.64). Urinary tract infections decreased from 4.5 (SD 3.2) to 1.1 (SD 1.36) per year and the number of hospitalizations was reduced from 2.8 (SD 3.2) to 0.9 (SD1) in the follow-up.

Conclusions In our population renal function remained the same, however surgical correction reduced the number of urinary tract infections and the number of hospitalizations. One of our limitations was the small number of patients, however prospective studies can be done to prove the relationship between surgery and the impact on these outcomes.

\section{Introducción}

La incidencia de reflujo vesicoureteral como parte de una complicación en el paciente llevado a trasplante renal es altamente variable, debido principalmente a la cirugía inicial y en el caso de la utilización de técnica antirreflujo, esta disminuye en un $10 \% .^{1-20}$
En el caso de las infecciones urinarias, son complicaciones frecuentes después del trasplante renal con una incidencia que oscila entre el $45 \%$ y el $72 \%$. Se han descrito múltiples factores de riesgo propios relacionados con la infección urinaria, así mismo, en el tratamiento urológico la presencia de un catéter doble J, estenosis ureteral, complicaciones técnicas con las anastomosis ureterales y el 
reflujo vesicoureteral contribuyen al riesgo de infecciones de vías urinarias en ese grupo de pacientes. ${ }^{2,15-20}$

En el estudio de infecciones urinarias recurrentes, se realiza la cistografía miccional para observar si presenta reflujo vesicoureteral, en el caso de hallar esa patología, los antibióticos profilácticos se pueden usar en reflujo de bajo grado es decir, I-II, mientras que el reflujo vesicoureteral de alto grado tiene indicación de tratamiento quirúrgico el cual se divide en dos tipos, la reconstrucción abierta y la endoscópica. $^{21,22}$ En cuanto a las complicaciones de la ureteroneocistostomía, se encuentran la alteración de estructuras anastomóticas, la necrosis ureteral y la persistencia de reflujo $1,9,11,15,18,21,23-33$

El objetivo de este trabajo, es describir los efectos clínicos de la corrección quirúrgica del reimplante vesicoureteral incluyendo la prevalencia de infecciones urinarias, hospitalizaciones previas y posterior a la reconstrucción de la vía urinaria en una institución de III nivel, entre el 2014 y el 2016.

\section{Materiales Y Métodos}

Es un estudio observacional, descriptivo de serie de expuestos, realizado en pacientes con trasplante renal que fueron sometidos a ureteronecistostomía endoscopica o abierta con técnica extravesical de espesor completo en una institución de III nivel, durante el periodo comprendido entre enero del 2014 a enero del 2016. Se revisaron las historias clínicas de la base de datos del grupo de trasplante de la Clínica Universitaria Colombia, así como también, se estudiaron las imágenes diagnósticas y los exámenes de laboratorio. En cuanto a los criterios de inclusión, se seleccionaron los pacientes registrados en la base de datos, mayores de edad trasplantados renales, con reflujo vesicoureteral demostrado en cistouretrografía miccional, llevados a reimplante ureterovesical entre el 2014 y el 2016 y se excluyeron los casos con información incompleta. Las variables estudiadas fueron la edad, el tipo de donante del trasplante renal, el grado de reflujo vesicoureteral previo y el abordaje quirúrgico de elección en el reimplante ureteral. Se realizó un análisis descriptivo utilizando medidas de frecuencia y tendencia central y se compararon las variables clínicas principales según el tipo de técnica quirúrgica usada. Para el análisis estadístico se usó el programa Statistics/Data Analysis v13.0 (Stata 13.0).

\section{Resultados}

En total se seleccionaron 16 pacientes para el estudio. El promedio de edad de la población de estudio fue de 43 años (D.E. $\pm 14,5$ ), el tipo de donante se obtiene que el cadavérico es el más frecuente con $16(87,5 \%)$ pacientes vs $2(12,5 \%)$ pacientes trasplantados con donante vivo. La prevalencia del grado de reflujo vesicoureteral previo a la corrección fue: en grado II 3 (25\%), grado III 5 (41,6\%), grado IV 3 (25\%) y en el caso de grado V 1 (8,3\%). El abordaje quirúrgico fue abierto con túnel antireflujo en un $68,7 \%$ (11) y endoscópico con inyección de dextranómero de ácido hialurónico en el 31,2\% (5) (-Tabla 1).

Respecto a las causas de la enfermedad renal terminal, el $50 \%$ (8) es de origen desconocido, el 12,5\% (2) debido a lupus eritematoso sistémico, $12,5 \%$ (2) secundario a la uropatía obstructiva, el 6,25\% (1) por enfermedad de riñón poliquístico y por último el $6,25 \%$ (1) correspondió a nefropatía diabética. Se encontró que la función renal posterior al reimplante muestra tendencia a la mejoría con niveles de creatinina pre en 1,66 (D.E. $\pm 1,19$ ) y post reimplante ureteral en 1,5 (D.E. $\pm 0,64$ ) $\mathrm{mg} / \mathrm{dl}$. Por otra parte, las infecciones urinarias disminuyeron de 4,5 (D.E. $\pm 3,8$ ) a 1,1 (D.E. $\pm 1,36$ ) por año y el número de hospitalizaciones se redujeron de 2,8 (D.E. \pm 3,2) a 0,9 (D.E. $\pm 1,28$ ) en el seguimiento (-Tabla 2). Del total de pacientes, el $87,5 \%$ (14) presentaron infecciones urinarias antes del reimplante ureteral y en el 50\% (8) de los casos se presentó posterior a ese.

En el análisis comparativo según el tipo de técnica quirúrgica empleada, se obtiene que, para el abordaje endoscópico, el promedio de edad fue de 44 (D.E. $\pm 9,13$ ) años, el 57,14\% (4) fueron pacientes con grado III de reflujo vesicoureteral. En el caso de los pacientes con abordaje en vía abierta, el promedio de edad fue de 42,54 (D.E. $\pm 16,89$ ) años y el grado II de reflujo vesicoureteral fue el más frecuente (3-60\%). En cuanto a las variables principales de estudio, se evidencia que la reducción en la creatinina se presenta en el grupo intervenido con abordaje endoscópico pasando de 1,75 (D.E. $\pm 1,44$ ) a 1,48 (D.E. $\pm 0,75$ ), el número de infecciones urinarias disminuyeron en ambos grupos (endóscopica: pre 4 (D.E. $\pm 3,31$ ) a post 1,18 (D.E. $\pm 1,40$ ); vía abierta pre 5,6 (D.E. $\pm 5,17$ ) a post 1 (D.E. $\pm 1,41$ ) y el número de hospitalizaciones se redujo en el grupo con abordaje vía abierta (5,2 - D.E. \pm 5,40 a 0,4 (D.E. $\pm 0,89$ ) (- Tabla 3).

Tabla 1 Descripción de características generales de la población del estudio

\begin{tabular}{|l|l|}
\hline Variable & Valor $(\boldsymbol{n}=16)$ \\
\hline Edad & $43(14,5)$ \\
\hline Media (D.E) & $11(68,7)$ \\
\hline Acceso quirúrgico (\%) & $5(31,2)$ \\
\hline Abierto & $3(25)$ \\
\hline Endoscópico & $5(41,6)$ \\
\hline Grado de reflujo vesicoureteral previo (\%) \\
\hline II & $3(25)$ \\
\hline III & $1(8,3)$ \\
\hline IV & $14(8,7)$ \\
\hline V & $2(12,5)$ \\
\hline Donante (\%)
\end{tabular}


Tabla 2 Resultados de variables pre y pos ureteroneocistostomía

\begin{tabular}{|l|l|l|}
\hline Características & $\begin{array}{l}\text { Pre* media } \\
\text { (D.E.) }\end{array}$ & $\begin{array}{l}\text { Pos* media } \\
\text { (D.E.) }\end{array}$ \\
\hline Creatinina & $1,66(1,19)$ & $1,50(0,64)$ \\
\hline $\begin{array}{l}\text { Infecciones de las vías } \\
\text { urinarias (IVU) }\end{array}$ & $4,5(3,8)$ & $1,1(1,36)$ \\
\hline $\begin{array}{l}\text { Hospitalizaciones } \\
\text { por IVU }\end{array}$ & $2,8(3,2)$ & $0,9(1,28)$ \\
\hline
\end{tabular}

*pre y pos reimplante ureteral.

Tabla 3 Comparación de las características de los pacientes según el abordaje quirúrgico

\begin{tabular}{|c|c|c|}
\hline \multirow[t]{2}{*}{ Características } & \multicolumn{2}{|c|}{ Abordaje quirúrgico } \\
\hline & Abierto & Endoscópico \\
\hline \multicolumn{3}{|l|}{ Edad } \\
\hline Media (D.E.) & $42,54(16,89)$ & $44(9,13)$ \\
\hline \multicolumn{3}{|c|}{ Grado de reflujo pre (\%) } \\
\hline II & 0 & $3(60,0)$ \\
\hline III & $4(57,14)$ & $1(20,0)$ \\
\hline IV & $2(28,57)$ & $1(20,0)$ \\
\hline V & $1(14,29)$ & 0 \\
\hline \multicolumn{3}{|l|}{ Donante (\%) } \\
\hline Cadavérico & $9(81,82)$ & $5(100,0)$ \\
\hline Vivo & $2(18,18)$ & 0 \\
\hline \multicolumn{3}{|c|}{ Creatinina - pre* (mg/dL) } \\
\hline Media (D.E.) & $1,75(1,44)$ & $1,45(0,28)$ \\
\hline \multicolumn{3}{|c|}{ Creatinina - pos* (mg/dL) } \\
\hline Media (D.E.) & $1,48(0,75)$ & $1,54(0,33)$ \\
\hline \multicolumn{3}{|c|}{ Número de IVU - pre* } \\
\hline Media (D.E.) & $4(3,31)$ & $5,6(5,17)$ \\
\hline \multicolumn{3}{|c|}{ Número de IVU - pos* } \\
\hline Media (D.E.) & $1,18(1,40)$ & $1(1,41)$ \\
\hline \multicolumn{3}{|c|}{ Número de hospitalizaciones por IVU - pre* } \\
\hline Media (D.E.) & $1,18(0,75)$ & $5,2(5,40)$ \\
\hline \multicolumn{3}{|c|}{ Número de hospitalizaciones por IVU - pos* } \\
\hline Media (D.E.) & $1,18(1,40)$ & $0,4(0,89)$ \\
\hline
\end{tabular}

Abreviaturas: IVU, infecciones vías urinarias.

*pre y pos reimplante ureteral.

\section{Discusión}

La probabilidad de reflujo vesicoureteral se incrementa con el tiempo después del trasplante, así mismo se ha asociado su incidencia con las diferentes técnicas quirúrgicas usadas, es por ello que expertos recomiendan la técnica con

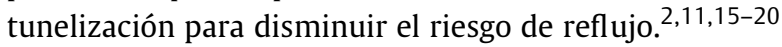

En el año 2007, se publicó la primera serie de casos de pacientes trasplantados con reflujo vesicoureteral manejados con deflux, se observó una resolución de la patología en la mayoría de los casos con una sola inyección y en cuatro pacientes mujeres que no mejoraron, finalmente requirieron reimplante ureteral abierto. ${ }^{5}$ Por otra parte, en el 2011, Pichler realizó un estudio con 19 pacientes trasplantados que sufrían más de tres infecciones al año, el reflujo se resolvió en un 57,9\% después de una inyección inicial y el 78,9\% después de dos tratamientos, también encontraron una reducción del número de infecciones por año de 4,89 a 1,31 casos. $^{5}$ En otro estudio se documentó que la tasa de éxito del procedimiento endoscópico estaba relacionada con el grado de reflujo vesicoureteral, en el $90 \%$ de los pacientes con grado I y II, se resolvió la enfermedad comparado con un 31\% de los casos con grado III y IV. Comparado con nuestro estudio se observó que los pacientes con reflujo vesicoureteral de menor grado (I y II) y sintomáticos eran candidatos al tratamiento endoscópico, mientras que los pacientes con mayor grado de enfermedad (III y IV) se eligió la vía abierta.

Por otra parte, existen algunos estudios que niegan la relación entre el reflujo vesicoureteral y las infecciones urinarias. Un estudio publicado en el 2009 comparaba a 15 trasplantados con reflujo y 22 sin reflujo vesicoureteral, cada paciente tenía por lo menos un episodio de IVU en el año anterior y dos o más episodios años después de la cirugía. No había diferencias entre el número de infecciones por año y niveles de creatinina en los dos grupos, sin embargo, se excluyeron los pacientes con reflujo vesicoureteral grado IV o V. ${ }^{1-6}$ En un estudio similar, Jung y colaboradores observaron a 75 pacientes trasplantados con reflujo vesicoureteral dentro de los cuales un $61 \%$ de los pacientes tenían grado IV y $\mathrm{V}$ versus sin reflujo. No se encontraron diferencias en la función renal o las infecciones de vías urinarias entre los dos grupos, no obstante, el seguimiento de este estudio fue menor a 1 año. ${ }^{1-6}$ Comparado con nuestro estudio, se documentó previo al manejo quirúrgico un promedio de 4,5 infecciones urinarias por año, con una tasa de hospitalización por esa causa de 2,8 . Una vez se realizó el reimplante ureteral, el número de infecciones urinarias disminuyó al 1,1, al igual que el número de hospitalizaciones a 0,9. En lo referente al nivel de creatinina, ella permaneció estable en algunos pacientes $\mathrm{y}$ en promedio ese paraclínico mejoró de $1.66 \mathrm{mg} / \mathrm{dL}$ (D.E. $\pm 1,19$ ) a $1.50 \mathrm{mg} / \mathrm{dL}$ (D.E. $\pm 0,64$ ) una vez se realizó la corrección de la enfermedad.

Se considera que el reflujo vesicoureteral en el riñón trasplantado es una enfermedad poco frecuente y está relacionada con la técnica utilizada en el momento del trasplante renal. Hay controversia si presentar esta condición aumenta el riesgo de infecciones urinarias y altera la función renal, dado que los estudios previos, tienen sesgos importantes en su ejecución, tales como no incluir a los altos grados de reflujo vesicoureteral o el tiempo de seguimiento. En el presente artículo, se describieron las características clínicas en este grupo de pacientes y el efecto de la corrección de esta patología en cuanto a la función renal y las infecciones urinarias. En nuestra población, los valores de creatinina permanecieron estables, sin embargo, el 
número de episodios de infecciones urinarias y de hospitalizaciones por esta causa disminuyeron posterior al reimplante ureterovesical. Una de nuestras limitaciones fue el pequeño número de pacientes, el carácter retrospectivo y por lo tanto, la posibilidad de que las infecciones urinarias se deban a otras causas diferentes al reflujo vesicoureteral.

Con esto se pretende, que en futuros estudios prospectivos, se evalúen las diferentes causas que existen del reflujo vesicoureteral en el paciente trasplantado, mencionando consideraciones importantes, tales como afectación de la calidad de vida, necesidad de tratamientos adicionales, complicaciones del manejo y costos.

\section{Conflictos de intereses}

Se declara que en el presente artículo no existen conflictos de intereses.

\section{Bibliografía}

1 Wein A, Kavoussi L, Novick A, Partin A, Peters G. Campbell Walsh Urología. Tomo 4. Décima edición. México DF: Editorial Panamericana; 2015

2 Lim J-H, Cho JH, Lee JH, et al. Risk factors for recurrent urinary tract infection in kidney transplant recipients. Transplant Proc 2013;45(04):1584-1589

3 Fishman JA. Infection in renal transplant recipients. Semin Nephrol 2007;27(04):445-461

4 Wu SW, Liu KS, Lin CK, et al. Community-acquired urinary tract infection in kidney transplantation: risk factors for bacteremia and recurrent infection. J Formos Med Assoc 2013;112(03): 138-143

5 Kayler L, Kang D, Molmenti E, Howard R. Kidney transplant ureteroneocystostomy techniques and complications: review of the literature. Transplant Proc 2010;42(05):1413-1420 10.1016/ j.transproceed.2010.04.016

6 Boonjindasup A, Smith A, Paramesh A, et al. A Rationale to Use Bladder Boari Flap Reconstruction for Late Kidney Transplant Ureteral Strictures. Urology 2016;89:144-149

7 He B, Bremner A, Han Y. Classification of ureteral stenosis and associated strategy for treatment after kidney transplant. Exp Clin Transplant 2013;11(02):122-127

8 Di Carlo HN, Darras FS. Urologic considerations and complications in kidney transplant recipients. Adv Chronic Kidney Dis 2015;22 (04):306-311

9 Karam G, Kälble T, Alcaraz A, Aki F, et al. Guidelineson Renal Transplantation. EuropeanAssociation of Urology; 2014

10 Jiang M, Gandikota N, Ames SA, Heiba S. Identification of urologic complications after kidney transplant. Am J Kidney Dis 2011;58 (01):150-153

11 Moreno CC, Mittal PK, Ghonge NP, Bhargava P, Heller MT. Imaging Complications of Renal Transplantation. Radiol Clin North Am 2016;54(02):235-249

12 Li Marzi V, Filocamo MT, Dattolo E, et al. The treatment of fistulae and ureteral stenosis after kidney transplantation. Transplant Proc 2005;37(06):2516-2517

13 Gonzalo Rodríguez V, Rivero Martínez MD, Trueba Arguiñarena J, Calleja Escudero J, Müller Arteaga C, Fernández del Busto E. Diagnóstico y tratamiento de las complicaciones uroógicas del trasplante renal. Actas Urol Esp 2006;30(06):619-625

14 Mitra S, Alangaden GJ. Recurrent urinary tract infections in kidney transplant recipients. Curr Infect Dis Rep 2011;13(06): 579-587

15 Hubert KC, Kokorowski PJ, Huang L, et al. New contralateral vesicoureteral reflux after unilateral ureteral reimplantation: predictive factors and clinical outcomes. J Urol 2014;191(02): 451-457

16 Ariza-Heredia EJ, Beam EN, Lesnick TG, Kremers WK, Cosio FG, Razonable RR. Urinary tract infections in kidney transplant recipients: role of gender, urologic abnormalities, and antimicrobial prophylaxis. Ann Transplant 2013;18:195-204

17 Ariza-Heredia EJ, Beam EN, Lesnick TG, Cosio FG, Kremers WK, Razonable RR. Impact of urinary tract infection on allograft function after kidney transplantation. Clin Transplant 2014;28 (06):683-690

18 Silva C, Afonso N, Macário F, Alves R, Mota A. Recurrent urinary tract infections in kidney transplant recipients. Transplant Proc 2013;45(03):1092-1095

19 Schumacher M, Vogt B, Frey F, Studer UEDH. ReUreterocystoneostomy Using The Lich-Gregor Technique inRenal Transplanted Patients Presenting With Vesico-ureteral Reflux And Recurrente Urinary Tract Infections. Eur Urol Suppl 2004;2:4

20 Harada H, Miura M, Takada N, Morooka K, Tanabe T, Seki T. Masaki Togashi TH. Vesicoureteral Reflux Into Kidney Allograft Has a Greater Negative Impact on Long-Term Graft Survival. J Urol 2008;179(04):697

21 Duty BD, Barry JM. Diagnosis and management of ureteral complications following renal transplantation. Asian J Urol 2015;2(04):202-207

22 Fontana I, Bertocchi M, Rossi AM, et al. Late ureteral stenosis after kidney transplantation: a single-center experience. Transplant Proc 2010;42(04):1174-1175

23 Silva A, Rodig N, Passerotti CP, et al. Risk factors for urinary tract infection after renal transplantation and its impact on graft function in children and young adults. J Urol 2010;184(04): $1462-1467$

24 Kmetec A, Buturović-Ponikvar J, Kandus A, Bren AF. The value of renal resistive index for the detection of vesicoureteral reflux in renal transplant recipients. Transplant Proc 2001;33(78):3385-3387

25 Kmetec A, Kaplan-Pavlovcic S, Kandus A, Bren AF. Surgical management of high-grade vesicoureteral reflux in renal transplant recipients. Transplant Proc 2001;33(7-8):3383-3384

26 Favi E, Spagnoletti G, Valentini AL, et al. Long-term clinical impact of vesicoureteral reflux in kidney transplantation. Transplant Proc 2009;41(04):1218-1220

27 Jung GO, Chun JM, Park JB, et al. Clinical significance of posttransplantation vesicoureteral reflux during short-term period after kidney transplantation. Transplant Proc 2008;40 (07):2339-2341

28 Saidi RF, Elias N, Hertl M, Kawai T, Cosimi AB, Ko DSC. Urinary reconstruction after kidney transplantation: pyeloureterostomy or ureteroneocystostomy. J Surg Res 2013;181(01):156-159

29 Hau HM, Tautenhahn HM, Schmelzle M, et al. Management of urologic complications in renal transplantation: a single-center experience. Transplant Proc 2014;46(05):1332-1339

30 Baston C, Harza M, Preda A, et al. Comparative urologic complications of ureteroneocystostomy in kidney transplantation: transvesical Leadbetter-Politano versus extravesical Lich-Gregoir technique. Transplant Proc 2014;46(01):176-179

31 Stoffel J, Sung R, Faerber G, Shields J, Wolf S, Huang Y. Mp85-19 Treatment of Transplant Ureter Strictures With Ureteral Dilation and Ureteroneocystostomy: Outcomes From a 14 Year Experience. J Urol 2015;193(04):e1074

32 Kayler L, Kang D, Molmenti E, Howard R. Kidney transplant ureteroneocystostomy techniques and complications: review of the literature. Transplant Proc 2010;42(05):1413-1420

33 Slagt IK, Dor FJ, Tran TC, et al. A randomized controlled trial comparing intravesical to extravesical ureteroneocystostomy in living donor kidney transplantation recipients. Kidney Int 2014; 85(02):471-477 